\title{
Bimetal single-molecule magnets supported on benzene with large magnetic anisotropy and unquenched orbital moment
}

\author{
Jianpei Xing, ${ }^{1}$ Xue Jiang $\odot,{ }^{1,}{ }^{*}$ Peng Wang $\odot,{ }^{2}$ and Jijun Zhao ${ }^{1}$ \\ ${ }^{1}$ Key Laboratory of Material Modification by Laser, Ion and Electron Beams (Dalian University of Technology), \\ Ministry of Education, Dalian 116024, China \\ ${ }^{2}$ School of Physical Science and Technology, Southwest University, Chongqing 400715, China
}

(Received 30 October 2020; revised 11 May 2021; accepted 12 May 2021; published 2 July 2021)

\begin{abstract}
Single-atom magnets, the ultimate limit of high-density magnetic storage, have attracted widespread attention over recent decades. However, they are far from being applicable as a storage medium because of their low magnetic stability. In this paper, we describe a strategy to induce huge magnetic anisotropy in "bimetal magnets" on a benzene (Bz) substrate based on the electron filling of $d$ orbitals. Our first-principles calculations reveal that $\mathrm{Os} X-\mathrm{Bz}(X=\mathrm{Fe}, \mathrm{Ru}$, and Os) exhibits high structural stability, large unquenched orbital moments $\left(1.55-1.59 \mu_{B}\right)$, and huge perpendicular magnetic anisotropy energy (MAE) above $54.8 \mathrm{meV}$. The synergistic effect of two transition metal components toward this huge MAE and preserved orbital moment is discussed in depth, which is mainly attributed to the $d$ - $d$ coupling induced energy level rearrangement. Our work may provide insights into the underlying physical mechanism and bimetal molecule magnet design.
\end{abstract}

DOI: 10.1103/PhysRevResearch.3.033005

\section{INTRODUCTION}

The continuous downscale of magnetic storage devices would finally reach the ultimate limit scale-one atom stores one bit of information. Hence, an urgent task is to design the new magnetic materials with high stability and long lifetime of the atom's magnetization. One of the critical parameters characterizing the magnetic stability is the magnetic anisotropy energy (MAE). MAE originates from the interaction of spin-orbit coupling (SOC) and ligand field (LF). In the past years, a major breakthrough on magnetic materials is the discovery of single-molecule magnets with large MAE, as a result of reduced dimensionality and symmetry. Many multimetallic and bimetallic single-molecule magnets, such as small metal clusters and dimers, were found to exhibit good performance in terms of maintaining a stable magnetic orientation, enabling a MAE value of up to several hundred $\mathrm{meV}$ [1-4]. The magnetic centers of single-molecule magnets are generally $3 d$ and $5 d$ transition metal (TM) atoms. Their advantages are the large spin moment in $3 d$ metal atoms and the strong SOC in the heavy $5 d$ atoms, respectively. As the smallest chemical species, transition metal dimers possess a significant energy barrier against the reorientation of their magnetization between the low-energy direction (dimer axis) and the high-energy direction (perpendicular to the dimer axis). Błoński and Hafner reported that the MAE of

\footnotetext{
*Corresponding author: jiangx@dlut.edu.cn

Published by the American Physical Society under the terms of the Creative Commons Attribution 4.0 International license. Further distribution of this work must maintain attribution to the author(s) and the published article's title, journal citation, and DOI.
}

the gas-phase homonuclear $\operatorname{Ir}_{2}$ dimer and the heteronuclear Ir-Co dimer can reach $70 \mathrm{meV}$ with large anisotropy of orbital moments of about $1.02 \mu_{B}$ and $1.2 \mu_{B}$ [5,6]. For the $\mathrm{Ir}_{2}$ dimer, Liang et al. found its MAE can be further enhanced up to $294 \mathrm{meV}$ by $F$ functionalization which drives orbital rearrangement [7]. Moreover, the formation of chemical bonds would quench orbital moment in multimetallic single-molecule magnets. For dimers, Fritsch et al. discovered that free $\mathrm{Fe}_{2}$ and $\mathrm{Co}_{2}$ dimers possess MAE above $30 \mathrm{meV}$ and large unquenched orbital moment anisotropy (OMA) of about $1.7 \mu_{B}$ and $1.67 \mu_{B}$, respectively [8]; Chen et al. found that the $\mathrm{Pt}_{2}$ dimer has a large OMA of about $2 \mu_{B}$ [9]. Niemeyer et al. demonstrated that the orbital moments are strongly quenched even for clusters of only three atoms [10].

Tuning the local environment of molecular magnets to produce an axial ligand field can have an important consequence on preserving the orbital moment as well as attaining the large MAE. Two-dimensional (2D) materials can be naturally utilized as supporting substrates to provide the appropriate ligand field. A variety of two-dimensional materials have been examined for a long time, such as Au [11], Pt [12], black phosphorus [13], graphyne [14], and graphene [15]. For example, the key magnetic parameters of transition dimers Os-Ru and $\mathrm{Co}_{2}$ adsorbing on pristine, single-vacancy (SV) or nitrogenized divacancy (NDV) graphene have been reported [15-17]. For the Os-Ru dimer absorbed on pristine (NDV) graphene, the orbital moments of the top Os atom have pronounced changes, which is from $1.44 \mu_{B}\left(1.29 \mu_{B}\right)$ to $-0.02 \mu_{B}\left(0.21 \mu_{B}\right)$. The corresponding MAEs are found to be $176 \mathrm{meV}$ [15] and $54 \mathrm{meV}$ [17], respectively. $\mathrm{Co}_{2}$ dimer absorption on SV graphene also preserves the OMA and MAE. The MAE is about $53 \mathrm{meV}$ and the orbital moments of the top Co atom along the (001) and (100) axis are about $2.1 \mu_{B}$ and $0.3 \mu_{B}$ [16]. To achieve single-molecule magnets 
with high magnetic stability, it is meaningful to pursue the optimal compositions of the transition metal dimers as well as the substrates.

In this study, the magnetic stability of a series of transition metal dimers adsorbed on benzene is explored using first-principles calculations. Benzene $(\mathrm{Bz})$ is the simplest and smallest organic unit with $D_{6 \mathrm{~h}}$ symmetry. Various transition metal dimers $\left(M_{1} M_{2}\right)$ have been considered by varying the combinations and configurations. After high-throughput screening of many $M_{1} M_{2}$ compositions, the $5 d$ element Os combined with same-group of transition metal atoms, labeled as $M_{1} M_{2}-\mathrm{Bz}\left(M_{1}=\mathrm{Os}, M_{2}=\mathrm{Fe}, \mathrm{Ru}\right.$, and Os), are picked up. They have strong SOC effect, partially occupied $d$ orbitals, large atomic magnetic moment, large anisotropic spin and orbital moments, and huge MAE. Both MAE and unquenched orbital moments are along the uniaxial direction. The synergistic effect of transition metal dimers and $\mathrm{Bz}$ substrates for regulating magnetic properties have also been further explained by either $p$ - $d$ hybridization between the lower $M_{2}$ metal with the $\mathrm{Bz}$ or $d$ - $d$ hybridization between $M_{1}$ and $M_{2}$ atoms. These results provide a feasible strategy to stabilize the magnetization in single-molecule magnets by achieving large MAE and unquenched orbital moment simultaneously.

\section{METHODS}

Using spin-polarized density functional theory (DFT) implemented in the VASP packages [18,19], first-principles calculations were performed to investigate the stability, electronic structures, and magnetic properties of $M_{1} M_{2}-\mathrm{Bz}$ systems ( $M=\mathrm{Os}, \mathrm{Ru}, \mathrm{Fe}, \mathrm{Ir}, \mathrm{Rh}$, and $\mathrm{Co})$. Based on the PerdewBurke-Ernzerhof (PBE) parametrization [20], the generalized gradient approximation (GGA) was used to describe the exchange and correlation effects. The projected augmented wave (PAW) [21] method was employed with an energy cutoff of $500 \mathrm{eV}$ to describe the electronic wave functions. The $M_{1} M_{2}-\mathrm{Bz}$ systems were placed in a large cubic box, ensuring a distance of no less than $20 \AA$ between neighboring periodic images to avoid any unphysical interaction. Geometry relaxation was performed until the force on each atom was less than $0.01 \mathrm{eV} / \AA$ and the total energy converged to $1 \times 10^{-7} \mathrm{eV}$. The $\Gamma$ point was used for the large cubic supercell of individual $M_{1} M_{2}-\mathrm{Bz}$.

The binding energy between $M_{1} M_{2}$ and $\mathrm{Bz}$ can be defined as

$$
E_{\mathrm{b}}=E_{M 1 M 2-\mathrm{Bz}}-E_{M 1 M 2}-E_{\mathrm{Bz}},
$$

where $E_{M_{1} M_{2}-\mathrm{Bz}}, E_{M 1 M 2}$, and $E_{\mathrm{Bz}}$ denote the total energies of the $M_{1} M_{2}-\mathrm{Bz}$, freestanding $M_{1} M_{2}$ dimer, and $\mathrm{Bz}$ molecule, respectively.

Based on the equilibrium configurations, the MAE was determined from the self-consistent calculation considering both SOC and magnetic noncollinearity, as follows:

$$
\mathrm{MAE}=\mathrm{E}(\|)-\mathrm{E}(\perp),
$$

where $E(\|)$ denotes the total energies for the magnetization direction along the $\mathrm{Bz}$ plane, while $E(\perp)$ represents the total energies for the magnetization direction parallel $(+z)$ or antiparallel $(-z)$ to the TM dimer. In our systems, we find that the $+z$ and $-z$ directions are identical and give the same results. For perpendicular magnetic storage device applications, a positive MAE corresponding to the out-of-plane easy magnetization direction is more valuable [22-24].

Moreover, the torque method developed by Wang et al. [25] was employed to conduct a thorough and quantitative analysis of the origin of the MAE of $M_{1} M_{2}-\mathrm{Bz}$ complexes. In the framework of the torque method [25], the MAE is expressed as

$$
E_{\mathrm{MAE}}=\sum_{i \in \mathrm{OCC}}\left\langle\psi_{i}\left|\frac{\partial H_{\mathrm{SO}}}{\partial \theta}\right| \psi_{i}\right\rangle_{\theta=45^{\circ}}
$$

Here, $\psi_{i}$ is the $i$ th relativistic eigenvector, and $H_{\text {SO }}$ is the SOC Hamiltonian. In this approach, the MAE is independent of the absolute accuracy of two total energy calculations. For the convenience of analysis, MAE was divided into contributions from three types of coupling, namely the majority spin states $(u u)$, the minority spin states $(d d)$, and also the cross-spin coupling $(u d+d u)$.

Based on the second order perturbation approach proposed by Wang, Wu, and Freeman [26], the MAE contributed by each atom can be approximately determined by matrix elements of the angular momentum operators $L_{z}$ and $L_{x}$, across the unoccupied $(u)$ and occupied $(o)$ states:

$$
\begin{aligned}
\operatorname{MAE} \approx & \xi_{n}{ }^{2} \sum_{o, u, \alpha, \beta}\left(2 \delta_{\alpha \beta}-1\right) \\
& \times\left(\frac{\left\langle o, \alpha\left|L_{z}\right| u, \beta\right\rangle^{2}}{E_{u, \alpha}-E_{o, \beta}}-\frac{\left\langle o, \alpha\left|L_{x}\right| u, \beta\right\rangle^{2}}{E_{u, \alpha}-E_{o, \beta}}\right) .
\end{aligned}
$$

Here, the SOC constant $\xi_{n}$ is an element dependent property where $n$ is the effective principal quantum number of the $d$ electron, which is the radial integral of the product of the SOC amplitude and the radical wave functions of the states $O$ and $u$. We have given a different matrix for each kind of element (including $\mathrm{Os}, \mathrm{Ru}, \mathrm{Fe}, \mathrm{C}$, and $\mathrm{H}$ ) involving the $M_{1} M_{2}-\mathrm{Bz}$ molecules. $\alpha$ and $\beta$ denote different spin channels. When $\alpha$ and $\beta$ are in the same spin channel, $\delta_{\alpha \beta}=1$; otherwise, $\delta_{\alpha \beta}=0$. We define

$$
P=\left\langle o, \alpha\left|L_{z}\right| u, \beta\right\rangle^{2}-\left\langle o, \alpha\left|L_{x}\right| u, \beta\right\rangle^{2} .
$$

Each $M_{1} M_{2}-\mathrm{Bz}$ molecule involves $3 d, 4 d$, and $5 d$ TM atoms. Hence, the strong correlation effect of the localized $d$ orbitals must be treated carefully. To examine the electron-electron correlation effect on MAE, we performed test calculations using the GGA $+U$ method [27,28]. In these TM based systems, a strong correlation among the localized $d$ orbitals is necessary. Thus, the Hubbard $U$ term was tested in the GGA $+U$ formulation of first-principles calculations [29,30]. An effective interaction parameter $U_{\text {eff }}$ is used to represent the value of $U-J$ in the subsequent discussion. For $\mathrm{Os}, \mathrm{Ru}$, and $\mathrm{Fe}$, the value of $U$ is typically about $1.5 \mathrm{eV}$. In our work, three values of $U_{\text {eff }}(0.5,1.5$, and 2.5$)$ were tested, and the results show that magnetic and orbital moment anisotropies do not vanish with the implementation of the $U_{\text {eff }}$ term, and increase slightly (see Table S1 of the Supplemental Material [31]). 
TABLE I. Equilibrium bond lengths of Os- $X$ and $X$-Bz, binding energy $\left(E_{b}\right)$, spin magnetic moments on TM atom, and MAE of Os $X$ $\mathrm{Bz}(X=\mathrm{Fe}, \mathrm{Ru}$, and $\mathrm{Os})$.

\begin{tabular}{lcccccc}
\hline \hline $\begin{array}{l}\text { Structure } \\
(\mathrm{Os} X-\mathrm{Bz})\end{array}$ & $\begin{array}{c}\text { Bond length } \\
(\mathrm{Os}-X)(\AA)\end{array}$ & $\begin{array}{c}\text { Bond length } \\
(X-C)(\AA)\end{array}$ & $\begin{array}{c}E_{b} \\
(\mathrm{eV})\end{array}$ & $\begin{array}{c}M_{\mathrm{Os}} \\
\left(\mu_{B}\right)\end{array}$ & $\begin{array}{c}M_{X} \\
\left(\mu_{B}\right)\end{array}$ & $\begin{array}{c}\mathrm{MAE} \\
(\mathrm{meV})\end{array}$ \\
\hline $\mathrm{OsFe}-\mathrm{Bz}$ & 2.06 & 2.08 & -1.81 & 1.519 & 0.252 & 67.2 \\
$\mathrm{OsRu}-\mathrm{Bz}$ & 2.18 & 2.23 & -2.56 & 1.565 & 0.168 & 58.5 \\
$\mathrm{Os}_{2}-\mathrm{Bz}$ & 2.21 & 2.24 & -2.84 & 1.492 & 0.173 & 54.83 \\
\hline
\end{tabular}

\section{RESULTS AND DISCUSSION}

As stated above, we use benzene as the substrate, and the two transition metal atoms are chosen from all the possible $3 d-5 d$ transition metals. After full relaxation, the magnetic moments and MAEs of a series of transition metal dimers adsorbed on benzene were determined by noncollinear DFT calculations. As listed in Table S2, the systems of $\mathrm{Fe}, \mathrm{Co}$, and $\mathrm{Ni}$ groups have large MAE. Especially, $\mathrm{Fe}$ and Co groups exhibit giant perpendicular MAE. For example, OsFe-Bz, OsRu-Bz, $\mathrm{Os}_{2}-\mathrm{Bz}, \mathrm{IrCo}-\mathrm{Bz}$, and $\mathrm{IrRh}-\mathrm{Bz}$ are about $67.20,58.5,54.83,81.53$, and $72.35 \mathrm{meV}$, respectively. Previously, IrCo-Bz has been studied by Xiao et al. and its MAE was about $248 \mathrm{meV}$ calculated by an all-electron fullpotential local-orbital scheme [32]. $M_{1} M_{2}$-Bz molecules by other groups have either a very small MAE or even no magnetism. For Ti and Mn groups, the HfTi-Bz and ReMn-Bz have small MAE of about 2.5 and $-5.94 \mathrm{meV}$ with fully quenched orbital angular momentum. $\mathrm{V}$ and $\mathrm{Cr}$ groups all behave as nonmagnetic. Hence, we will mainly focus on the Fe group to discuss the synergistic effect between two TM atoms in producing the huge MAE in the following contents.

The atomic structures, electronic structures, and magnetic properties of Os-Fe, Os-Ru, and $\mathrm{Os}_{2}$ dimers absorbed on $\mathrm{Bz}$ are systematically investigated and the related parameters are listed in Table I. First, three possible high-symmetry structures are built. As a representative, Fig. 1 illustrates the structural model and checks the relative energies of configurations I $\left(\Delta E_{1}\right)$ and II $\left(\Delta E_{2}\right)$ of OsFe-Bz. For Os-Ru dimer and $\mathrm{Os}_{2}$ dimers, $\Delta E_{1}$ are 1.08 and $1.38 \mathrm{eV}$, and $\Delta E_{2}$ are $0.94 \mathrm{eV}$ and $1.26 \mathrm{eV}$, respectively. These relative energy calculations clearly prove that all the $\mathrm{Fe}$ group dimers prefer an upright position above the center of $\mathrm{Bz}$ with $C_{6 \mathrm{v}}$ symmetry. Besides, we consider two possible situations in configuration III: Os near Bz and away from Bz. The energy calculations show that Os atom prefers to stay away from the $\mathrm{Bz}$ in configuration III. Specifically, we will use OsX-Bz ( $X=\mathrm{Fe}, \mathrm{Ru}$, and $\mathrm{Os})$ to denote the $\mathrm{Os} X$ dimer adsorbed on Bz upright, where $X$ is the bottom atom nearest to the benzene. We can see that, naturally, the bond lengths of Os- $X$ and $X-C$ increase gradually from $\mathrm{OsFe}-\mathrm{Bz}$ to $\mathrm{Os}_{2}-\mathrm{Bz}$ because the reducibility to release electrons of $\mathrm{Fe}$ is stronger than that of $\mathrm{Ru}$ and Os according to the activity series of metals [33]. The binding energies of $\mathrm{Os} X-\mathrm{Bz}$ systems defined by Eq. (1) are in the range -1.81 to $-2.84 \mathrm{eV}$ (Table I), implying the exothermic formation of these dimer-benzene complexes.

To gain deep insight into the interactions of Os- $X$ and $X-\mathrm{Bz}$, the partial density of states (PDOS) of OsFe-Bz, OsRu-Bz, and $\mathrm{Os}_{2}-\mathrm{Bz}$ are calculated and displayed in Fig. 2. Clearly, in
(I)

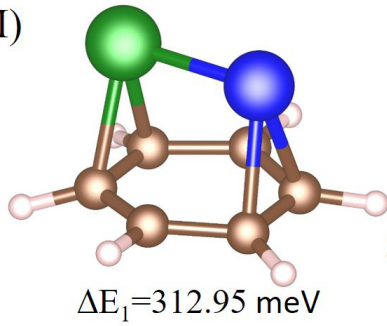

(II)

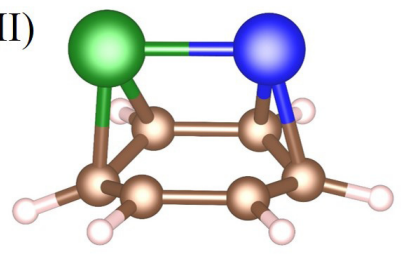

$\Delta \mathrm{E}_{2}=553.34 \mathrm{meV}$

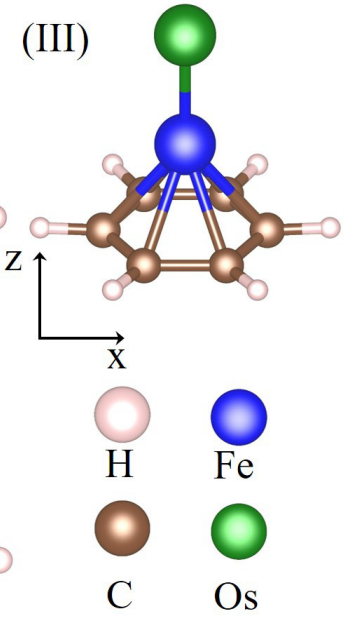

FIG. 1. I, II, III are three possible high-symmetry structures for Os $X$-Bz complexes. Here, $\Delta E_{1}$ and $\Delta E_{2}$ are the energy difference of (I) - (III) and (II) - (III) of OsFe-Bz, respectively. The positive value represents that structure III possesses lowest energy which can be as the ground state structure.

the OsFe-Bz system, the interaction between two TM atoms includes two types of strong hybridization: $5 d_{x 2-y 2}-3 d_{x 2-y 2}$, $5 d_{x z / y z}-3 d_{x z / y z}$, and $5 d_{z 2}-3 d_{z 2}$. The corresponding bonding and antibonding states can be denoted as $\pi / \pi^{*}, \alpha / \alpha^{*}$, and $\sigma / \sigma^{*}$, respectively. The $\pi / \pi^{*}$ and $\sigma / \sigma^{*}$ are fully occupied in the spin-up channels, whereas both spin-up and spin-down channels of $\alpha / \alpha^{*}$ and the spin-down channels of $\pi / \pi^{*}$ and $\sigma / \sigma^{*}$ are partially occupied. In OsRu-Bz and $\mathrm{Os}_{2}-\mathrm{Bz}$, only $\sigma / \sigma^{*}$ and $\alpha / \alpha^{*}$ states exist and $\alpha / \alpha^{*}$ are very weak. Meanwhile, the spin-up and spin-down channels of $\sigma / \sigma^{*}$ states are fully and partially occupied, respectively. Regarding the $X$ - $C$ bonds $(X=\mathrm{Fe}, \mathrm{Ru}$, and $\mathrm{Os}$ ), only $p$ - $d$ hybridization exists at a deep energy level, i.e., $d_{x z / y z}-p_{z}$. Furthermore, Mulliken population analysis in the CASTEP package [34] reveals that the Fe (Ru,

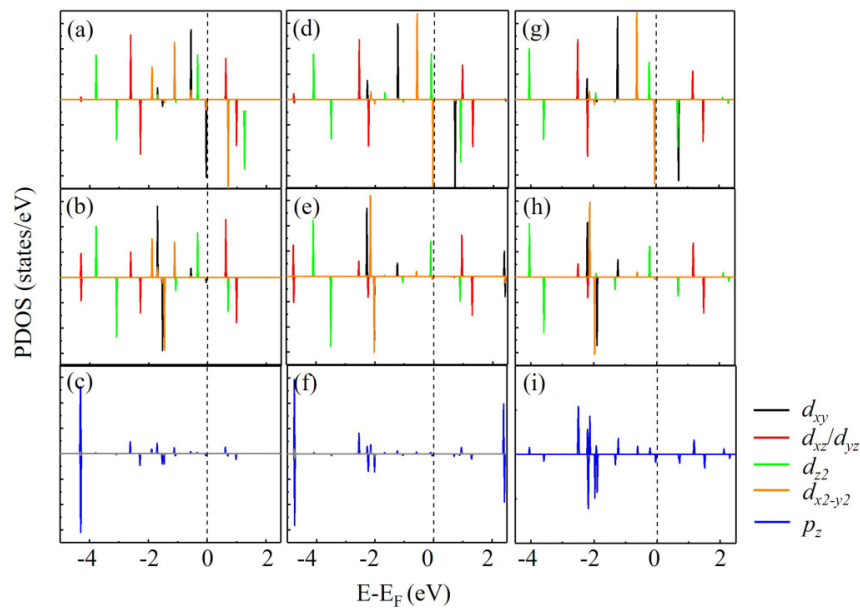

FIG. 2. PDOS of TM atoms of OsFe-Bz and OsRu-Bz. (a)-(c) PDOS of Os, Fe, and Bz for OsFe-Bz. (d)-(f) PDOS of Os, Ru, and $\mathrm{Bz}$ for OsRu-Bz. (g)-(i) PDOS of Os upper, Os bottom, and Bz for $\mathrm{Os}_{2}-\mathrm{Bz}$. The Fermi level $\left(E_{\mathrm{F}}\right)$ is set to zero. 
Os) atom shares nearly $0.24(0.09,0.17)$ electrons with the top Os atom and gives $1.63(0.92,0.91)$ electrons to Bz. Totally, the $\mathrm{Fe}$ atom contributes nearly two electrons $\left(1.87 e^{-}\right)$to the surrounding atoms, exhibiting a $\mathrm{Fe}^{2+}$ chemical state in this molecule. We can see that $\mathrm{Fe}$ shares more electrons with the top Os atom compared with $\mathrm{Ru}$ and Os because $\mathrm{Fe}$ has smaller electronegativity (1.83) than $\mathrm{Ru}(2.2)$ and Os (2.2), as given by the Pauling scale. Additionally, bond population analyzed by CASTEP [34] shows that the bond order of the Os-Fe bond $(0.77)$ is stronger than that of Os- $\mathrm{Ru}(0.46)$ and Os-Os (0.63). Such strong bonding interactions make a major contribution to the structural stability of $\mathrm{Os} X-\mathrm{Bz}$ complexes.

Our spin-polarized DFT calculations reveal the coexistence of large spin and orbital magnetic moments in Os $X$-Bz systems. First, the top $5 d$ transition metal Os atom provides the majority of spin magnetic moments as listed in Table I, which are about $1.52 \mu_{B}, 1.57 \mu_{B}$, and $1.49 \mu_{B}$ for OsFe-Bz, $\mathrm{OsRu}-\mathrm{Bz}$, and $\mathrm{Os}_{2}-\mathrm{Bz}$, respectively. This can be more clearly visualized from the spin charge density in Fig. S1. From the PDOS of TM atoms in Fig. 2, we can understand the origin of the magnetic moment in those systems. For OsFe-Bz complexes, the magnetic moment of Os mainly comes from the partially occupied $d_{z 2}$ and $d_{x 2-y 2}$ orbitals (about $0.5 \mu_{B}$ and $1 \mu_{B}$, respectively). The $\mathrm{Fe}$ atom only contributes about $0.25 \mu_{B}$ through $d_{z 2}$ orbitals. Similarly, in Figs. 2(d) and 2(g), the $d_{x y}$ orbital of the top Os atom provides about $1 \mu_{B}$, and $d_{z 2}$ contributes $0.51 \mu_{B}$ and $0.44 \mu_{B}$ for $\mathrm{OsRu}-\mathrm{Bz}$ and $\mathrm{Os}_{2}-\mathrm{Bz}$, respectively. Here, the Ru atom in OsRu-Bz and the bottom Os atom in $\mathrm{Os}_{2}$-Bz only provide about $0.17 \mu_{B}$ through the $d_{z 2}$ orbital. Second, the orbital angular momentums of Os $X$-Bz are largely preserved, as shown in Table S3. The orbital moments reach up to $1.55 \mu_{B}$ and $1.59 \mu_{B}$ along the dimer axis while the in-plane direction is only about $0.15 \mu_{B}$ and $0.2 \mu_{B}$, respectively. Besides, we observed the difference of spin moment between the out-of-plane and in-plane directions in a few systems. The anisotropy of spin moment is originating from the SOC induced reordering of the eigenstates close to the Fermi level, which have been explained in detail in the previous work. For our systems, it is larger in $\mathrm{Os} X-\mathrm{Bz}$ than in the $\mathrm{Fe}$ and $\mathrm{Ru}$ based systems. Meanwhile, the larger spin moment is found along the out-of-plane (easy-axis) orientation. One can see that the large spin magnetic moment of the top $5 d$ TM atom and the unquenched orbital moment on the TM atoms are protected very well. Thus, this low coordination environment provided by the bottom TM really plays a positive role in preserving the free-atom-like properties of the $5 d \mathrm{TM}$ element, as proved by our DFT calculations. A similar large magnetic moment was also observed for the Os containing molecules [35,36].

Considering SOC together with magnetic noncollinearity, the MAE was evaluated by both self-consistent calculations and the torque method. The $\mathrm{Os} X-\mathrm{Bz}(X=\mathrm{Fe}, \mathrm{Ru}$, and $\mathrm{Os})$ exhibit large MAE values of about 67.2 (59.92), 58.5 (55.54), and $54.83 \mathrm{meV}(52.9 \mathrm{meV})$, respectively, whereas the values from torque model calculations are given in brackets. Obviously, the PAW method used in our study has also been supported by the torque method. The two methods obtain the same results. With the help of the torque method, we decompose the MAE into three coupling terms with respect to the Fermi level (Table II). As seen in Fig. 3, the coupling
TABLE II. Contributions of different spin channels, including majority spin states $(u u)$, minority spin states $(d d)$, and cross spin states $(u d+d u)$; total MAE of $\mathrm{Os} X-\mathrm{Bz}(X=\mathrm{Fe}, \mathrm{Ru})$ given by torque calculations.

\begin{tabular}{lcccc}
\hline \hline Structure & $u u(\mathrm{meV})$ & $d d(\mathrm{meV})$ & $u d+d u(\mathrm{meV})$ & Total $(\mathrm{meV})$ \\
\hline OsFe-Bz & -48.18 & 99.30 & 8.79 & 59.92 \\
OsRu-Bz & -41.98 & 99.98 & -2.46 & 55.54 \\
Os $_{2}-\mathrm{Bz}$ & -33.29 & 80.82 & 5.35 & 52.90 \\
\hline \hline
\end{tabular}

between occupied and unoccupied spin-down $d$ states $(d d$ term) provides the major contribution to the positive MAE compared with other couplings near the Fermi level in those three systems. Combined with the PDOS analysis (Fig. 2), it can be concluded that the large positive MAE of Os $X$-Bz mainly comes from the $d d$ term, which is composed of the spin-down orbitals of occupied $5 d_{x y} / d_{x 2-y 2}$ and unoccupied $5 d_{x 2-y 2} / d_{x y}$. Those orbitals have a small energy difference of about $0.71 \mathrm{eV}$ and are coupled by a large coupling coefficient $P=4$, which is obtain by Eq. (5). As stated by Edström [37], a large MAE might appear if there are many occupied and unoccupied states with energies very close to the Fermi energy. Correspondingly, it is demonstrated directly in Eq. (4) that a smaller energy difference between occupied and unoccupied states in the denominator is generally more beneficial for obtaining a large MAE. Besides, the same picture can be also applied to interpret the small MAE of other groups such as Ti and Mn groups. As shown in Fig. S2, Near the Fermi level, the small MAE values of HfTi-Bz ( $2.5 \mathrm{meV})$ and ReMn-Bz $(-5.94 \mathrm{meV})$ are only contributed by the partial occupied $d$ orbitals $\left(d_{x z} / d_{y z}\right.$ and $\left.d_{x y} / d_{x z}\right)$ with the coupling coefficient $P=1$ and -1 , respectively.

To further clarify the composition effect, the difference of MAE between OsFe-Bz and $\mathrm{OsRu}-\mathrm{Bz}$ is discussed. As stated above, the energy difference between occupied and unoccupied states near the Fermi level also plays a key role in determining the strength of magnetic anisotropy. All three coupling terms are given in Table II. Among them, we find that the $u d+d u$ term is the key factor. The $u d+$ $d u$ term of $\mathrm{OsFe}-\mathrm{Bz}$ is $8.79 \mathrm{meV}$, notably larger than that of OsRu-Bz of $-2.46 \mathrm{meV}$. The orbital energy levels and the coupling coefficients of $u d+d u$ terms are listed in Table III. It can be clearly seen that the smaller $\Delta E$ between occupied and unoccupied states of $\mathrm{Os}$ atom in OsFe-Bz accounts for the greater SOC and MAE with regard to

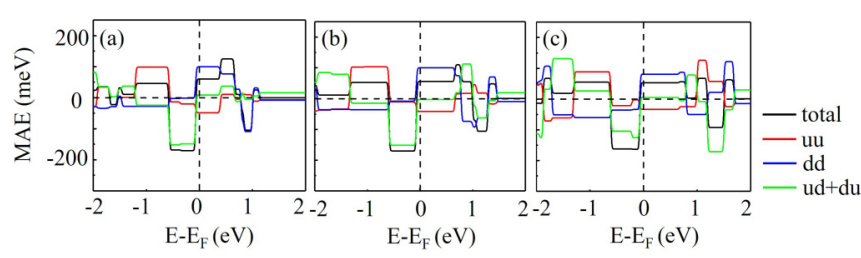

FIG. 3. Fermi level dependent total and decomposed MAEs of $\mathrm{OsFe}-\mathrm{Bz}(\mathrm{a}), \mathrm{OsRu}-\mathrm{Bz}(\mathrm{b})$, and $\mathrm{Os}_{2}-\mathrm{Bz}$ (c). Here, majority (red line), minority (blue line), cross (green line) represent the coupling between spin-up channels, spin-down channels, and spin-up with spin-down channels, respectively. The Fermi level $\left(E_{\mathrm{F}}\right)$ is set to zero. 
TABLE III. Orbital energy levels, energy level difference $(\Delta E)$, and coupling coefficient $(P)$ of $\mathrm{Os} X-\mathrm{Bz}(X=\mathrm{Fe}, \mathrm{Ru})$; Fermi level $\left(E_{\mathrm{F}}\right)$ is set to zero.

\begin{tabular}{lcccccccc}
\hline \hline & \multicolumn{3}{c}{ OsFe-Bz } & & \multicolumn{3}{c}{ OsRu-Bz } \\
\cline { 2 - 4 } \cline { 6 - 8 }$o / u$ & $o^{+}$ & $u^{-}$ & $\Delta E$ & & $o^{+}$ & $u^{-}$ & $\Delta E$ & $P$ \\
\hline$d_{z 2} / d_{y z}$ & -0.34 & 0.98 & 1.32 & & -0.10 & 1.30 & 1.4 & 3 \\
$d_{x y} / d_{x z}$ & -0.56 & 0.98 & 1.54 & & -1.24 & 1.30 & 2.54 & 1 \\
$d_{x 2-y 2} / d_{y z}$ & -0.58 & 0.98 & 1.56 & & -0.58 & 1.30 & 1.88 & 1 \\
$d_{x y} / d_{x 2-y 2}$ & -0.56 & 0.70 & 1.26 & & -0.58 & 0.70 & 1.28 & -4 \\
\hline \hline
\end{tabular}

OsRu-Bz. Such an energy level difference is caused by the coordination environment, which is determined by the bottom transition metal atom $X$. All the results suggest that a lighter base atom may modulate the electron structure of the top metal atom more effectively. Coincidentally, this phenomenon has also been observed in systems with group 9 elements: IrCo-Bz and IrRh-Bz. The MAE of $\mathrm{IrCo}-\mathrm{Bz}$ is $\sim 81.53 \mathrm{meV}$, somewhat larger than the $72.35 \mathrm{meV}$ of IrRh-Bz.

To illuminate the role of $3 d, 4 d, 5 d$ transition metal atoms and Bz, Table S3 presents the $M_{1}-M_{2}$ bond lengths, spin and orbital magnetic moments on TM atoms, and MAE given by noncollinearity self-consistent (NSC) method and torque method of free homonuclear Os, Fe, Ru dimers $\left(T M_{2}\right)$ and supported on $\mathrm{Bz}\left(T M_{2}-\mathrm{Bz}\right)$, free heteronuclear Os-Fe dimers, Os-Ru dimers, and single $\mathrm{Os}, \mathrm{Fe}, \mathrm{Ru}$ adatoms on $\mathrm{Bz}$ (TM-Bz). Previously, the MAE values of $\mathrm{Fe}_{2}, \mathrm{Ru}_{2}$, and $\mathrm{Os}_{2}$ dimers have been reported by Błoński and Hafners [6]. Our results for freestanding $\mathrm{Fe}_{2}$ and $\mathrm{Ru}_{2}$ dimers are generally consistent with their results in structure and physical properties, while the MAE value of $\mathrm{Os}_{2}$ disagrees (see Table S3).

As shown in Fig. 4(a), when comparing the freestanding $\mathrm{Os}_{2}\left(\mathrm{Fe}_{2}, \mathrm{Ru}_{2}\right)$ and $\mathrm{Os}_{2}-\mathrm{Bz}\left(\mathrm{Fe}_{2}-\mathrm{Bz}, \mathrm{Ru}_{2}-\mathrm{Bz}\right)$, or freestanding Os-Ru (Os-Fe) and OsRu-Bz (OsFe-Bz) the ligand field provided by $\mathrm{Bz}$ plays an important role in fixing the easy magnetization direction, regardless of whether it is a homonuclear or heteronuclear system. Moreover, the OMA in Os $X-\mathrm{Bz}$ systems is enhanced up to three times that of free dimers. In particular, when an Os-Fe dimer is anchored on $\mathrm{Bz}$, the spin anisotropy and OMA increase concurrently, thus obtaining a large MAE of $67.2 \mathrm{meV}$. As displayed in Fig. 4(b), the upper atom has two roles in a $M_{1} M_{2}$-Bz system. First, it induces the out-of-plane easy magnetization direction, as demonstrated by the results of Os-Bz $(\mathrm{Ru}-\mathrm{Bz}, \mathrm{Fe}-\mathrm{Bz})$ and $\mathrm{Os}_{2}-\mathrm{Bz}\left(\mathrm{Ru}_{2}-\mathrm{Bz}\right.$, $\mathrm{Fe}_{2}-\mathrm{Bz}$ ). Second, it also provides a strong SOC effect when

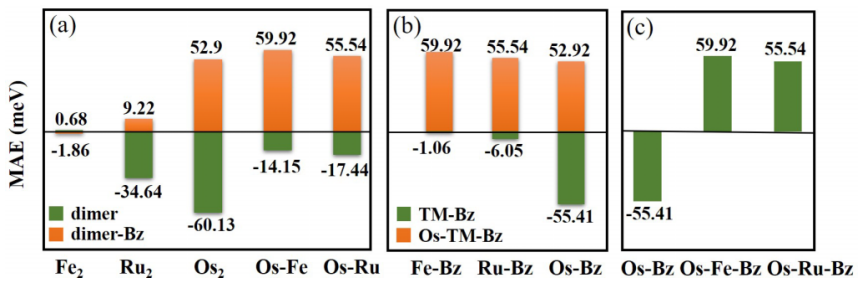

FIG. 4. MAE values of free dimers (homonuclear and heteronuclear dimers) and dimers or single atoms absorbed on Bz. a $5 d$ element is used. For example, when the top Fe atom in $\mathrm{Fe}_{2}-\mathrm{Bz}$ is replaced by $\mathrm{Os}$, the MAE increases significantly from -1.79 to $67.2 \mathrm{meV}$. For $\mathrm{Ru}_{2}-\mathrm{Bz}$ and $\mathrm{OsRu}-\mathrm{Bz}$, the MAE also increases from 9.22 to $58.5 \mathrm{meV}$. Similar to the above discussions about spin and orbital magnetic moments, the bottom metal atom in the Os-Bz and OsFe-Bz (OsRu-Bz) complexes weakens the influence of the ligand field on the $5 d$ TM atom [Fig. 4(c)]. This effect preserves the strong SOC strength of the top atom and makes it behave as a free atom.

According to previous experimental works, many similar molecules have already been synthesized by laser vaporization source, such as $\mathrm{Fe}_{\mathrm{n}} \mathrm{Bz}_{\mathrm{m}}, \mathrm{Ni}_{\mathrm{n}} \mathrm{Bz}_{\mathrm{m}}, \mathrm{Rh}_{\mathrm{n}} \mathrm{Bz}_{\mathrm{m}}, \mathrm{Co}_{\mathrm{n}} \mathrm{Bz}_{\mathrm{m}}$, and $\mathrm{Pt}_{\mathrm{n}} \mathrm{Bz}_{\mathrm{m}}$ [38-43]. Their magnetic recording properties have not been investigated, but highly anticipated. Although there is no evidence for the preparation of our proposed $M_{1} M_{2} \mathrm{Bz}$, we still believe they would be achieved using the same method later.

Presumably for these single-molecule magnets to be useful in magnetic storage devices, we have also investigated their magnetic stability on supporting bases. We put these bimetal $\mathrm{Os} X-\mathrm{Bz}$ molecules on the representative $2 \mathrm{D}$ surfaces, such as $h$-BN, black phosphorus (BP), and graphene. To simulate the interactions between supporting bases and Os $X-\mathrm{Bz}$ molecules, the $6 \times 6$ supercell for $h$-BN and graphene, and $4 \times 4$ supercell for BP were constructed, which are large enough to avoid interactions among dimers in the neighboring cells. Fortunately, one can see that the Os $X-\mathrm{Bz}$ molecules will not dissociate into adatoms when placed on $h$-BN, BP, and graphene (Fig. S3). In addition, the large magnetic moments of our studied Os $X-\mathrm{Bz}$ molecules are also preserved well in $h$-BN and BP (Table S4).

In our studied metal-benzene complexes, the strong coupling between transition metal atoms through $d$ - $d$ direct exchange interaction lead to the large MAE of $\sim 54 \mathrm{meV}$, which could provide a kind of feasible building blocks for designing magnetic recording devices. Together with MAE, exchange interaction is also the critical point to determine the preferred direction of the magnetization. Clearly, the exchange interaction between the different molecule magnets is rather weak, which can be negligible. To investigate the exchange interactions in the molecule magnets, we have calculated the energy difference between a spin-parallel and a spin-antiparallel state. The values are shown in Table S5, which is $180 \mathrm{meV}$ for OsRu-Bz $\left(\mathrm{Os}_{2}-\mathrm{Bz}\right)$ and $137 \mathrm{meV}$ for OsFe-Bz, respectively. The corresponding exchange coupling parameters are estimated to be 300 and $153 \mathrm{meV}$ for the $\mathrm{OsRu}-\mathrm{Bz}$ and $\mathrm{Os}_{2}-\mathrm{Bz}$ systems. One can see that the exchange interactions between two magnetic ions is strong enough to resist a thermally induced spin flipping.

\section{CONCLUSIONS}

To summarize, we have investigated the stability and magnetic properties of transition metal dimers adsorbed on benzene using systematical first-principles calculations. Positioning the dimer on the center of a carbon hexagon, arranged upright with the lighter atom nearest to the benzene, is the optimal configuration in terms of both stability and magnetic properties. When the $\mathrm{Os} X(X=\mathrm{Fe}, \mathrm{Ru}$, and Os) dimer is supported on Bz, the large orbital moment anisotropy of top Os atom can be achieved owing to the unquenching orbital 
angular momentum. Large MAEs above $54 \mathrm{meV}$ are obtained in $\mathrm{Os} X-\mathrm{Bz}$ systems. Furthermore, the origin of large MAE of $\mathrm{Os} X$-Bz complexes has been in depth understood and the roles of each component have been identified.

In the Os $X-\mathrm{Bz}$ complexes, the upper Os atom plays an important role in producing large MAE values when it is protected well by the lower transition metal $X$ atom with strong intra-atomic exchange integrals. Compared with $4 d$ or $5 d$ atoms, $3 d$ atoms in the lower metal site display better performance in terms of manipulating the electronic structure of the upper atom near the Fermi level. Such difference is due to stronger $d$ - $d$ direct exchange interaction between them than $4 d / 5 d$ atoms-based systems; the corresponding energy level shifts have been presented in the paper. The benzene substrate not only provides support for the dimer, but also fixes its easy magnetization axis to the perpendicular direction. Furthermore, the OsX-Bz molecules arranged on the bases ( $h$-BN, black phosphorus) still show good performance- that is, their structural stability and large magnetic moments are well retained. Our study provides a further understanding of the underlying physics about the magnetic anisotropy mechanism in bimetal single-molecule magnets. It may give some guidance about the design of multicenter molecular magnets.

\section{ACKNOWLEDGMENTS}

This work was supported by the National Natural Science Foundation of China (Grants No. 11874097 and No. 91961204) and the Fundamental Research Funds for the Central Universities of China (Grant No. DUT19LK12). We acknowledge the Xinghai scholar project of Dalian University of Technology and the project of Dalian Youth Science and Technology Star (Grant No. 2017RQ012). The authors also acknowledge the Supercomputing Center of Dalian University of Technology for providing the computing resources.
[1] P. Boński, S. Dennler, and J. Hafner, Strong spin-orbit effects in small Pt clusters: Geometric structure, magnetic isomers and anisotropy, J. Chem. Phys. 134, 034107 (2011).

[2] H. Mokkath and Junais, Nanometer size $3 d-4 d$ and $3 d-5 d$ substitutional clusters: Promising candidates for magnetic storageapplications, J. Magn. Magn. Mater. 334, 31 (2013).

[3] X. Liang, X. Wu, X. Huang, Y. Su, and J. Hu, Magnetic anisotropy of small $\mathrm{Ir}_{\mathrm{n}}$ clusters $(\mathrm{n}=2-5)$, J. Cluster Sci. 27, 935 (2016).

[4] V. Chandrasekhar and B. Murugesapandian, Phosphorussupported ligands for the assembly of multimetal architectures, Acc. Chem. Res. 42, 1047 (2010).

[5] P. Błońsk and J. Hafner, Magnetic anisotropy of heteronuclear dimers in the gas phase and supported on graphene: Relativistic density-functional calculations, J. Phys.: Condens. Matter 26, 146002 (2014).

[6] P. Błońsk and J. Hafner, Magnetic anisotropy of transitionmetal dimers: Density functional calculations, Phys. Rev. B 79, 224418 (2009).

[7] X. Liang, X. Wu, J. Hu, J. Zhao, and X. C. Zeng, Large magnetic anisotropy in chemically engineered iridium dimer, Commun. Phys. 1, 74 (2018).

[8] D. Fritsch, K. Koepernik, M. Richter, and H. Eschrig, Transition metal dimers as potential molecular magnets: A challenge to computational chemistry, J. Comput. Chem. 29, 2210 (2008).

[9] J. Chen, H. Cui, G. Wang, T. Zhou, H. Chen, and H. Yuan, Deposition morphology and magnetism of Co, Pt adatoms and small CoPt adclusters on Ni (100) substrate, J. Cluster Sci. 27, 947 (2016).

[10] M. Niemeyer, K. Hirsch, V. Zamudio-Bayer, A. Langenberg, M. Vogel, M. Kossick, C. Ebrecht, K. Egashira, A. Terasaki, T. Möller, B. v. Issendorff, and J. T. Lau, Spin Coupling and Orbital Angular Momentum Quenching in Free Iron Clusters, Phys. Rev. Lett. 108, 057201 (2012).

[11] A. Antal, B. Lazarovits, L. Udvardi, L. Szunyogh, B. Újfalussy, and P. Weinberger, First principles calculation of spin-interactions and magnetic ground states of $\mathrm{Cr}$ trimers on Au(111), Phys. Rev. B 77, 174429 (2008).
[12] C. Etz, J. Zabloudil, P. Weinberger, and E. Vedmedenko, Magnetic properties of single atoms of $\mathrm{Fe}$ and $\mathrm{Co}$ on $\operatorname{Ir}(111)$ and Pt(111), Phys. Rev. B 77, 184425 (2008).

[13] H. Liu, Y. Du, Y. Deng, and P. D. Ye, Semiconducting black phosphorus: Synthesis, transport properties and electronic applications, Chem. Soc. Rev. 44, 2732 (2015).

[14] Y. Zhang, G. Zhu, J. Lu, Z. Guo, and J. Cao, Graphyne as a promising substrate for high density magnetic storage bits, RSC Adv. 5, 87841 (2015).

[15] K. Zhang, Y. Li, Y. Liu, and Y. Zhu, Protecting quantum anomalous Hall state from thermal fluctuation via the giant magnetic anisotropy of Os-based dimers, Phys. Chem. Chem. Phys. 20, 28169 (2018).

[16] H. C. Kandpal, K. Koepernik, and M. Richter, Strong magnetic anisotropy of chemically bound Co dimers in a graphene sheet, Phys. Rev. B 86, 235430 (2012).

[17] J. $\mathrm{Hu}$ and $\mathrm{R}$. Wu, Giant magnetic anisotropy of transitionmetal dimers on defected graphene, Nano Lett. 14, 1853 (2014).

[18] G. Kresse and J. Furthmüller, Efficient iterative schemes for ab initio total-energy calculations using a plane-wave basis set, Phys. Rev. B 54, 11169 (1996).

[19] G. Kresse and J. Furthmüller, Efficiency of ab-initio total energy calculations for metals and semiconductors using a plane-wave basis set, Comput. Mater. Sci. 6, 15 (1996).

[20] J. P. Perdew, K. Burke, and M. Ernzerhof, Generalized Gradient Approximation Made Simple, Phys. Rev. Lett. 78, 1396(E) (1997).

[21] P. E. Blöchl, Projector augmented-wave method, Phys. Rev. B 50, 17953 (1994).

[22] P. Wang, X. Jiang, J. Hu, X. Huang, and J. Zhao, Giant magnetic anisotropy of a $5 \mathrm{~d}$ transition metal decorated two-dimensional polyphthalocyanine framework, J. Mater. Chem. C 4, 2147 (2016).

[23] P. Wang, X. Jiang, J. Hu, and J. Zhao, Chemically engineering magnetic anisotropy of 2D metalloporphyrin, Adv. Sci. 4, 1700019 (2017). 
[24] J. Xing, P. Wang, Z. Jiang, X. Jiang, Y. Wang, and J. Zhao, Rational design of 2D organic magnets with giant magnetic anisotropy based on two-coordinate 5d transition metals, APL Mater. 8, 071105 (2020).

[25] X. Wang, R. Wu, D. Wang, and A. J. Freeman, Torque method for the theoretical determination of magnetocrystalline anisotropy, Phys. Rev. B 54, 61 (1996).

[26] D. Wang, R. Wu, and A. J. Freeman, First-principles theory of surface magnetocrystalline anisotropy and the diatomic-pair model, Phys. Rev. B 47, 14932 (1993).

[27] S. L. Dudarev, G. A. Botton, S. Y. Savrasov, C. J. Humphreys, and A. P. Sutton, Electron-energy-loss spectra and the structural stability of nickel oxide: An LSDA+U study, Phys. Rev. B 57, 1505 (1998).

[28] V. I. Anisimov, I. Solovyev, M. Korotin, M. Czyżyk, and G. Sawatzky, Density-functional theory and NiO photoemission spectra, Phys. Rev. B 48, 16929 (1993).

[29] B. Su and N. Li, Electronic and magnetic properties of $5 \mathrm{~d}$ transition metal atoms doped blue phosphorene: First-principles study, J. Magn. Magn. Mater. 469, 236 (2019).

[30] M. Zhang, H.-m. Guo, J. Lv, and H.-s. Wu, Electronic and magnetic properties of $5 \mathrm{~d}$ transition metal substitution doping monolayer antimonene: Within GGA and GGA+ U framework, Appl. Surf. Sci. 508, 145197 (2020).

[31] See Supplemental Material at http://link.aps.org/supplemental/ 10.1103/PhysRevResearch.3.033005 for the spin-polarized charge density of OsX-Bz; PDOS of HfTi-Bz, TaV-Bz, WCr$\mathrm{Bz}$, and ReMn-Bz; the configuration and magnetic moments of Os $X$-Bz absorbed on bases; MAE of Os $X$-Bz with different $U_{\text {eff }}$; magnetic moments and MAE of $M_{1} M_{2}$-Bz molecules; magnetic properties of free and $\mathrm{Bz}$ absorbed homonuclear, heteronuclear dimers and single atom; and the exchange interaction parameter $(J)$ of $\mathrm{Os} X$-Bz.

[32] R. Xiao, M. D. Kuz'Min, K. Koepernik, and M. Richter, CoIrcarbon complexes with magnetic anisotropies larger than 0.2 eV: A density-functional-theory prediction, Appl. Phys. Lett. 97, 232501 (2010).

[33] W. F. Kieffer, The activity series of the metals, J. Chem. Educ. 27, 659 (1950).
[34] S. J. Clark, M. D. Segall, C. J. Pickard, P. J. Hasnip, M. I. Probert, K. Refson, and M. C. Payne, First principles methods using CASTEP, Z. Kristallogr. - Cryst. Mater. 220, 567 (2005).

[35] J. Qu and J. Hu, Engineering giant magnetic anisotropy in single-molecule magnets by dimerizing heavy transition-metal atoms, Appl. Phys. Express 11, 055201 (2018).

[36] L. Qu, J. Zhang, and K. Xu, Structural, electronic and magnetic properties of $5 \mathrm{~d}$ transition metal mediated benzene adsorption on graphene: A first-principles study, Synth. Met. 209, 225 (2015).

[37] A. Edström, Theoretical and Computational Studies on the Physics of Applied Magnetism: Magnetocrystalline Anisotropy of Transition Metal Magnets and Magnetic Effects in Elastic Electron Scattering (Acta Universitatis Upsaliensis, Uppsala, 2016).

[38] M. Gerhards, O. Thomas, J. Nilles, W.-J. Zheng, and K. Bowen Jr., Cobalt-benzene cluster anions: Mass spectrometry and negative ion photoelectron spectroscopy, J. Chem. Phys. 116, 10247 (2002).

[39] W. Zheng, S. N. Eustis, X. Li, J. M. Nilles, O. C. Thomas, K H. Bowen, and A. K. Kandalam, Photoelectron spectroscopic study of iron-benzene cluster anions, Chem. Phys. Lett. 462 , 35 (2008).

[40] D. W. Ball, Z. H. Kafafi, R. H. Hauge, and J. L. Margrave, Reactions of iron atoms with benzene and cyclohexadiene in argon matrixes: Iron-benzene complexes and photolytic dehydrogenation of cyclohexadiene, J. Am. Chem. Soc. 108, 6621 (1986).

[41] W. Zheng, J. M. Nilles, O. C. Thomas, and K. H. Bowen Jr., Photoelectron spectroscopy of nickel-benzene cluster anions, J. Chem. Phys. 122, 044306 (2005).

[42] G. Lüttgens, N. Pontius, C. Friedrich, R. Klingeler, P. Bechthold, M. Neeb, and W. Eberhardt, Chemisorption of benzene on metal dimer anions: A study by photoelectron detachment spectroscopy, J. Chem. Phys. 114, 8414 (2001).

[43] C. Berg, M. Beyer, T. Schindler, G. Niedner-Schatteburg, and V. E. Bondybey, Reactions of benzene with rhodium cluster cations: Competition between chemisorption and physisorption, J. Chem. Phys. 104, 7940 (1996). 